\title{
CERN comes out again on top
}

\author{
With the discovery of the electroweak bosons ( $W^{ \pm}$and $\left.Z^{0}\right)$ in the bag, CERN now announces the \\ discovery of the quark called top. What will come next?
}

THE Matthew principle - "to him that hath shall be given" - is working in favour of CERN, the European high-energy physics laboratory at Geneva, and of the UA1 collaboration which, at the end of last year, announced the discovery of the $\mathrm{W}^{ \pm}$ and $Z^{0}$ particles which mediate the electroweak interaction. Last week, the same 80 -strong collaboration, under the leadership of Carlo Rubbia, announced the discovery of the missing sixth quark, called top, long-predicted but hitherto elusive. By doing so, they have put yet another cap on the electroweak theory while restoring a seemly symmetry to the evolving picture of quarks as the elementary constituents of the material Universe.

The new development at CERN follows almost exactly along the lines expected (and described, for example, by $\mathrm{Dr} F$. Close in his comment on the electroweak bosons, see Nature 303, 656; 1983). The source of the sixth quark is a charged boson, $\mathrm{W}^{+}$or $\mathrm{W}^{-}$, first recognized at CERN by their decay into an electron (with electrical charge of the same sign), with excess momentum carried away by a neutrino. Events of this kind accumulated at CERN in the past two years have amply confirmed that the mass of the $\mathrm{W}^{ \pm}$particles is that predicted by the electroweak theory, the equivalent of $82 \pm 2 \mathrm{GeV}$. The neutral member of the trio of heavy bosons, the $Z^{0}$, is less frequently produced (by a factor of about 10) in the proton-antiproton collisions at CERN, has a greater mass (to the tune of an extra $12 \mathrm{GeV}$ ) and is chiefly recognizable by its decay into a pair of electrons, positive and negative.

Although the chief decay path for the $\mathrm{W}^{ \pm}$bosons is that by which their existence was first recognized, it has from the outset been accepted that decay schemes leading to the production of quarks should be recognizable alternatives. Briefly, a $\mathrm{W}^{+}$ particle should be capable of yielding a top and the antimatter version of a bottom quark. ( $\mathbf{W}^{-}$would then yield anti-top and anti-bottom.) For the past two years, there has been general agreement on the way in which these particles could be recognized. The bottom quark (or anti-quark) would itself decay into a narrow jet of nuclear matter - pi-mesons for example. And the top quark, with a greater mass, would first decay into bottom and then yield another jet of particles, this time less tightly collimated. Since the first evidence for $\mathrm{W}^{ \pm}$ particles began to accumulate at CERN, people have been wondering whether some of the events recorded by UAl were signs of decay of this kind. Six events have now been unambiguously identified as the decay of $\mathrm{W}^{ \pm}$into top and bottom; the mass of top, estimated at $40 \mathrm{GeV}$, remains substantially uncertain.

For the time being, however, the proof that top exists is enough to be going on with. In the simplest terms, the asymmetry that has now been removed is that between the set of known electron-like particles and the set of quarks. For reasons which are frankly not understood, the natural world contains not just one material lepton, the electron (and its anti-particle, the positron), but two others, the muon and the tauon (each with its oppositely charged anti-particle). With each of these three leptons is associated a distinctive neutrino, recognizably different in the mechanisms by which they interact with matter but, on present form, not otherwise distinguishable - they have no electrical charge and no mass. But neutrinos are, like electrons, true leptons - they are involved symmetrically with electrons, muons and tauons in the working of the weak nuclear interaction (as in beta decay).

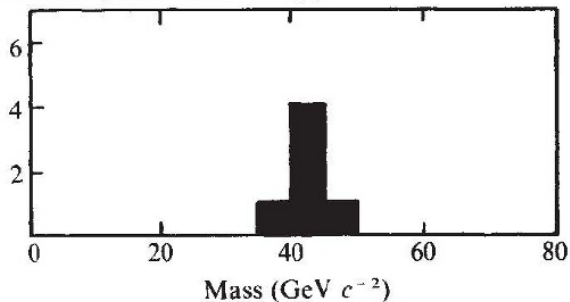

Distribution of measured top quark mass.

The idea that quarks should also come in pairs, and that there should be as many pairs of quarks as there are pairs of leptons, is more an act of faith than a consequence of theoretical expectation. To be sure, if the world is symmetrical in this way, it is possible to build neater theories, more symmetrical than would otherwise be the case. But that is merely a sign that, in its foundations, theoretical physics remains Pythagorean.

Phenomenologically, the need for symmetry has nevertheless been urgent since the late 1940s. The recognition of the difference between the pi-meson and the muon first raised the puzzle of the apparently superfluous lepton. The discovery (in cosmic rays) at the same time of a new kind of hadronic (nuclear) matter, called strange because that is what it was, set the scene for Gillman's radical proposal that mesons such as the pi-meson, but also the strange particles themselves, are pairs of quarks - the pi-meson is a pair called up and down for example. But nucleons, such as protons and neutrons, and other baryons, are combinations of three quarks - the proton, for example, is two $u p$ quarks and one down. The partner of strange, discovered only in 1975, is charm. Evidence for bottom, also known as beauty, was found in 1977 in the protonproton collisions arranged at Fermilab, where a meson whose mass exceeds the equivalent of $9.4 \mathrm{GeV}$ was surmised to be a bound state of bottom and anti-bottom.

The quark called top (and also, sometimes, truth) is thus the missing member of the series. Its appearance has been expected for some time, but is no less welcome to the closet-Pythagoreans on that account. What will, in the short term, matter more is that the steady refinement of the mass now on the cards should make possible a degree of certainty about the nature of some still disputed hadronic particles and resonances. While the electroweak theory itself has been further confirmed, CERN and its UAl collaboration have provided a more stringent test both of theories of quantum chromodynamics (theories of the strong nuclear interaction) and of Grand Unified Theories (which would roll that together with the electroweak theory but not - yet - with gravitation). Only time will tell whether the outcome is any confirmation of some version or another surprise - yet another pair of leptons or quarks, for example.

Inevitably, the question will arise in Britain whether the discovery of the top quark at the collaborative high-energy physics laboratory will bear on the decision, now delegated to a committee under Sir John Kendrew, on whether Britain should continue to collaborate. The arguments run both ways. The discovery of top means that CERN's list of unattained achievements has been reduced by one, but at the same time the laboratory's reputation for success has been enhanced. It is, however, unlikely that the committee's recommendations will be determined by scalp-counting of this kind, while highenergy physicists will properly draw attention to the need, now, for the careful understanding of the relationships between the six quarks that will come only from more careful measurements of the decay schemes now recognized, and of the alternatives still to be found.

John Maddox 